\title{
Quel lectorat pour la géographie entre 1650 et 1780 ?
}

\section{Nicolas Verdier}

\section{CpenEdition}

\section{Journals}

Édition électronique

URL : https://journals.openedition.org/geohist/1015

DOI : 10.4000/geohist.1015

ISSN : 2264-2617

Éditeur

Association française de la Revue de géographie historique

\section{Référence électronique}

Nicolas Verdier, «Quel lectorat pour la géographie entre 1650 et 1780 ? », Revue de géographie

historique [En ligne], 17-18 | 2020, mis en ligne le 03 novembre 2020, consulté le 12 juin 2021. URL

http://journals.openedition.org/geohist/1015; DOI : https://doi.org/10.4000/geohist.1015

Ce document a été généré automatiquement le 12 juin 2021.

\section{cc) $(1) \ominus$}

Ce(tte) œuvre est mise à disposition selon les termes de la Licence Creative Commons Attribution -

Pas d'Utilisation Commerciale - Pas de Modification 4.0 International. 


\title{
Quel lectorat pour la géographie entre 1650 et 1780 ?
}

\author{
Nicolas Verdier
}

\section{Introduction}

1 Comprendre la place que la géographie occupe dans le concert des savoirs du XVIII ${ }^{\mathrm{e}}$ siècle reste difficile. De nombreux éléments offrent des indices de la fabrication d'un champ de savoirs géographiques, depuis l'affirmation de titres de géographes, qu'ils soient d'un prince, d'une institution comme la ville de Paris, ou du Roi, jusqu'à la multiplication des dictionnaires de géographie. Il faudra cependant attendre la fin du $\mathrm{XIX}^{\mathrm{e}}$ siècle, et la mise en place d'une discipline universitaire, pour qu'un champ scientifique au sens fort se mette en place (Rhein, 1982; Bourdieu, 1997). Mais limiter l'investigation aux seuls producteurs de ces savoirs, et de leur reconnaissance par des autorités - quelles qu'elles soient - c'est en grande partie perdre de vue la question de la réception, et au-delà, des usages. C'est oublier qu'une science à des auteurs, mais qu'elle a aussi un public. Il semble possible en s'appuyant sur les réflexions développées par David Edgerton de travailler plus avant cette question des usages (Edgerton, 1998). Ce faisant, à la question récurrente d'une histoire des sciences désormais classique, - et en fait dépassée - "se trompaient-ils? » il semble intéressant ici de préférer - sans exclusive - «qu'en faisait-on? " C'est pourquoi, après avoir travaillé la question des usages de la carte, puis celle de la relation entre carte et géographie dans différents textes (Verdier, 2015a et 2015b), il convient maintenant de passer à la question du public de la géographie et de ses appropriations des savoirs. Il ne s'agit pas dans un texte court de prétendre répondre à ces vastes interrogations, mais plus simplement de suivre quelques pistes relatives à l'histoire du livre de géographie entre la fin du XVII ${ }^{\mathrm{e}}$ et le début du XIXe siècle (Martin, 1983 ; Martin et Chartier, 1982-1986 ; Chartier, 1992). 


\section{Les livres de géographie dans le marché du livre}

2 Passons dans un premier temps par une tentative de mesure de l'extension du savoir géographique durant cette période. On s'intéressera ici à l'une des échelles d'observations les plus vastes, c'est-à-dire le catalogue de la Bibliothèque nationale de France tel qu'il existe aujourd'hui. On pourrait longuement discuter de la pertinence de ce choix, en se demandant d'où viennent les collections, en prenant garde à leur représentativité par rapport aux livres réellement diffusés, voire en tenant compte des tirages. On arriverait cependant très rapidement à la conclusion que si cet outil est imparfait, il nous offre des informations impossibles à réunir autrement (Balayé, 1988). Il convient donc d'être conscient des limites de la source sans pour autant l'abandonner. Dans cette étude, le critère choisi pour interroger le catalogue de la Bibliothèque nationale est la présence des mots «géographie(s)» et "géographique(s)» dans leurs titres (quelles qu'en soient les graphies), en partant du principe que si tous les livres de géographie ne contiennent pas ce mot dans leur titre, en revanche le fait d'utiliser le mot sous-entend une volonté forte de s'agréger au géographique. Dans cette période pré-disciplinaire, ce marqueur semble un élément pertinent de définition de l'un des pôles du savoir (Nordman, 1988 ; Rey, 1994).

Livres dont le titre renvoie à la Géographie dans le catalogue de la Bibliothèque Nationale de France

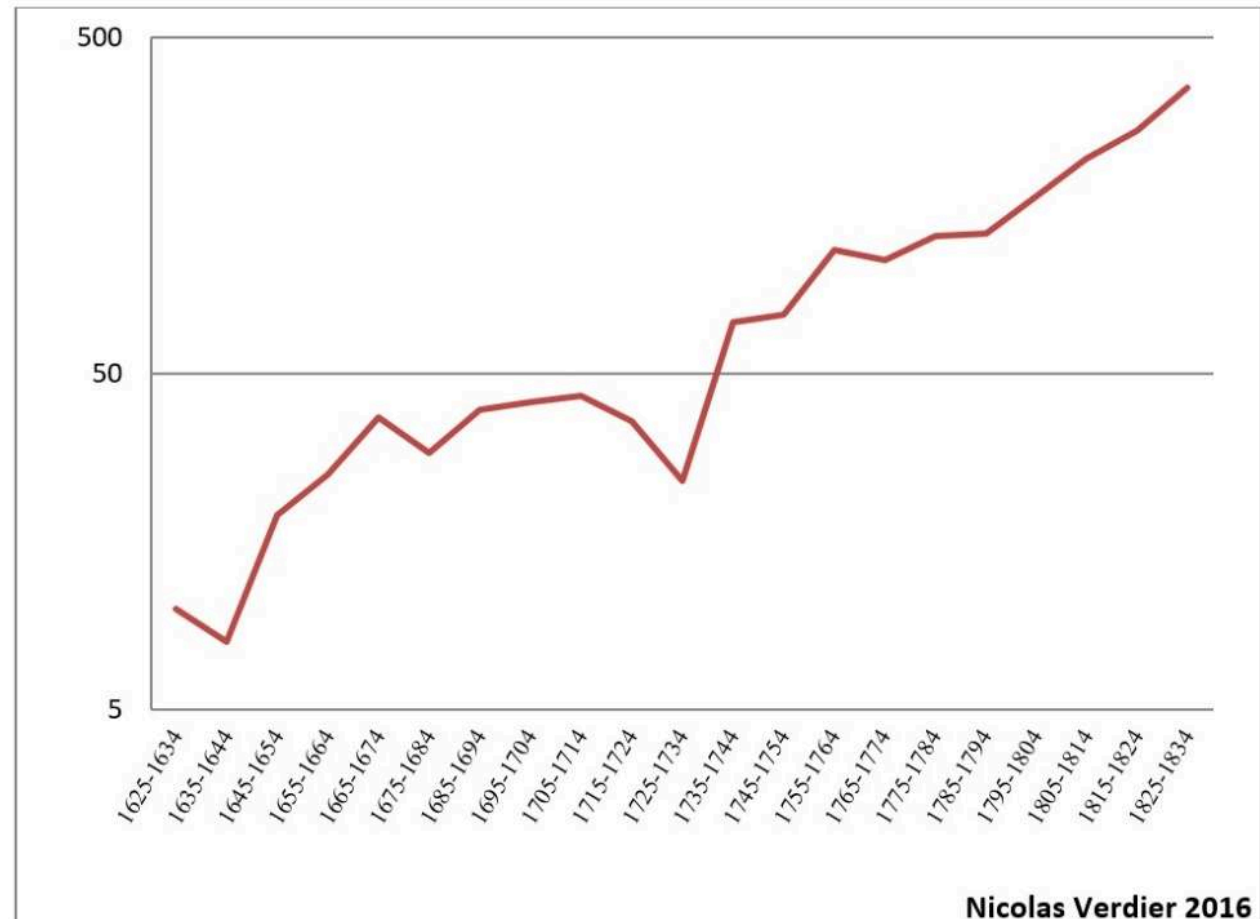

Sur les 1874 livres correspondant au critère, la simple observation du graphique semilogarithmique permet de déterminer que deux moments organisent la croissance des livres de géographie. Le premier, de 1620 à 1720 est celui d'une lente augmentation de leur nombre, qui passe d'une dizaine à une quarantaine d'ouvrages par décennie. Après un affaiblissement marqué durant la décennie 1725-1735, on assiste à la mise en place d'une croissance soutenue jusqu'au XIX ${ }^{e}$ siècle, avec le passage de 70 à 350 ouvrages par décennie de 1735 à 1835 . On a donc une multiplication par quatre de 1625 à 1725 , puis 
par cinq de 1735 à 1835. Cette croissance quasi exponentielle de la publication d'ouvrages de géographie entre 1630 et 1830 est un indice fort de la diffusion de ce savoir dans la société. Relativisons cependant: cette belle croissance doit être rapprochée de la production française dans son ensemble. On passe alors de 0,9 à 4,9\%o du total des livres en français ; croissance forte donc, mais relative $(0,89 \%$ ontre 1625 et $1654 ; 1,87 \%$ ontre 1655 et $1684 ; 2,22 \%$ ontre 1685 et $1714 ; 1,68 \%$ o entre 1715 et $1744 ; 3,6 \%$ entre 1745 et $1774 ; 2,04 \%$ o entre 1775 et 1804 et 4,92\%o entre 1805 et 1834 ). Qu'en est-il si l'on observe ce qui se passe à une autre échelle soit celle des lecteurs? Il est possible d'aborder cette question de deux façons successives. La première s'intéressera au discours que les géographes tiennent sur leur lectorat, que ce soit dans leurs livres ou dans les autres textes qu'ils font paraître : c'est la question du public visée qui sera ici posée. La seconde essayera de recomposer le marché de la géographie, non plus dans le discours des auteurs, mais dans l'un des lieux de sa réception qu'est la bibliothèque privée. Ce sont donc des traces matérielles de la réception de la géographie que l'on tentera ici de mettre au jour.

\section{L'injonction géographique}

4 Prenons ici quatre textes qui se répartissent entre 1678 à 1716 pour les premières éditions, mais qui, ayant d'autres éditions - parfois remaniées -, permettent d'étendre la période jusqu'en 1768 . Nous couvrons ainsi une période qui va du dernier tiers du $\mathrm{XVII}^{\mathrm{e}}$ siècle au deuxième tiers du XVIII ${ }^{\mathrm{e}}$ siècle. Ces ouvrages sont celui d'Augustin Lubin, Le Mercure géographique, celui de Gilles de Launay, La cosmographie aisée et celui des Sanson, l'Introduction à la Géographie, et pour finir, la Méthode pour étudier la géographie de Lenglet du Fresnoy.

5 L'ouvrage d'Augustin Lubin a bénéficié un temps d'une forte reconnaissance, même s'il n'est édité qu'une fois (Dainville, 1964). Dès 1680 le Dictionnaire françois de Richelet cite Lubin comme une référence (Richelet, 1680, $2^{\mathrm{e}}$ p., 43-44), et l'éloge du père Augustin Lubin publié dans le Journal des Sçavans de 1695 (Journal des Sçavans, 1695, 220-222), posent le Mercure Géographique comme un texte majeur. En 1704, la seconde édition du Dictionnaire historique et Critique de Bayle offrira de longues citations du Mercure géographique (Bayle, 1704, t. 3, 2794). Le livre passe cependant rapidement au second plan et après les années 1720 , il n'est plus cité que dans les rééditions des dictionnaires, voire dans la série sans limite d'emprunts, lors de la fabrication de nouveaux dictionnaires.

6 Lubin qui s'intéresse pour l'essentiel aux cartes géographiques plus qu'à la géographie n'en définit pas moins le public de ce savoir : celui-ci correspond aux trois géographies qui coexistent à ses yeux. Celles-ci sont définies par leur rapport à trois périodes historiques (Lubin, 1678, 8).

La Géographie ancienne Vetus, la plus belle \& la plus sçavante, sans laquelle il est presque impossible de conserver la mémoire des belles actions [...]. Les théologiens ont grand sujet de la désirer pour la parfaite intelligence de la théologie positive. La seconde Géographie que l'on appelle du moyen temps medii temporis, qui traite de l'état de la terre [au moyen âge, n'est qu'un] discours sans cartes [...]. La troisième Géographie, appellée nouvelle, qui represente l'état des Royaumes des derniers temps [...], principalement nécessaire aux personnes qui par leurs emplois, sont obligez de traiter avec les étrangers : utile à ceux qui font des voyages, \& agreable aux amateurs de l'histoire du siècle \& aux curieux de nouvelles (Lubin, 1678, 10-11). 
7 Le public tel qu'il est défini par Lubin se réduit donc à des théologiens (groupe dans lequel il se comprend), pour la géographie ancienne, et à des personnes qui «par leur emplois sont obligez de traiter avec les étrangers ", voire à ceux qui voyagent, et pour finir aux amateurs d'histoire et aux curieux des nouvelles, pour la géographie nouvelle. La géographie du moyen âge ne semble pas avoir pris une importance suffisante pour que l'auteur en décrive le public. Quant à la géographie nouvelle, on remarquera que Lubin n'est pas dupe de sa description puisqu'à l'article Via il met en doute l'usage possible des cartes de géographie pour voyager : «quelqu'un de mes amis s'étant muni de la Carte d'un Voyage, qu'il alloit faire, il m'assura à son retour n'avoir trouvé ni les noms, ni les distances des lieux marquez dans cette Carte, si bien qu'il ne faut pas trop s'y fier » (Lubin, 1678, 235-236). Dans cet ouvrage, le lien entre la géographie et son public reste fragile.

8 La cosmographie aisée... (Launay, 1681) est publiée trois ans après le livre de Lubin. Écrite par Gilles de Launay, elle ne laissera que peu de traces dans les histoires de la géographie. L'ouvrage est rare et ne semble pas avoir été particulièrement lu. Cela n'empêche pas Launay d'essayer de définir un public. Il est, cette fois, plus précis en même temps que beaucoup plus large que Lubin :

le moindre Soldat ou Marchand qui a voyagé se raille de ceux qui l'ignorent [la Géographie] ; \& il n'y a aucun employ dans la vie civile où elle ne soit nécessaire ; elle sert aux Politiques à gouverner leurs Etats, aux Prélats à gouverner leurs Eglises, aux Capitaines à faire le guerre, aux Négotians à faire le commerce, \& universellement à tous les hommes, pour se représenter tout ce qui se fait sur le surface de la terre (Launay, 1681, Avis au lecteur, 1-2, n.p.).

Pour résumer, la géographie est utile à tous : soldats, marchands, emplois civils, acteurs politiques, religieux et militaires, négociants, et pour finir tous les hommes. Son utilité est en outre générale puisque dans le cadre de la culture classique elle « est nécessaire pour l'intelligence de l'Histoire, en faisant voir où se sont passées les plus fameuses actions des Héros de l'Antiquité... » (Launay, 1681, Avis au lecteur, 3-4, n.p.). On retrouve ici la fameuse formule de "Géographie œil de l'histoire ", qui commence à s'imposer à ce moment (Nordman, 1998).

10 En 1681, le troisième ouvrage, de Guillaume Sanson, va aussitôt être considéré comme un texte majeur. Il va connaître au moins sept rééditions avec une succession d'aménagements. La dernière édition, retravaillée par le petit-fils de Nicolas Sanson, Gilles Robert de Vaugondy, est publiée en 1743. Les Sanson (s), même s'ils vont rapidement être critiqué pour le maintien de fonds de cartes datés, conserveront un statut à part, de rénovateur français de la géographie. Nicolas Sanson est encore régulièrement appelé le «Père de la Cartographie » (Broc, 1970), ce qui correspond à ce que son fils Guillaume écrit dans le livre. Il est vrai que, dans ses différentes éditions, l'Introduction à la Géographie est un livre beaucoup plus courant que les deux précédents, puisqu'on le retrouve dans la plupart des bibliothèques publiques. Sanson est probablement celui qui développe le plus la description de ce public potentiel :

Non seulement les Poëtes, les Philosophes \& les Historiens, ne peuvent négliger la Géographie sans tomber dans des Beveuës qui sont nullement excusables, mais mesme il n'y a aucun Employ dans la Vie Civile ou cette Science ne soit pas nécessaire.

Les Souverains \& les parfaits Politiques ne peuvent sans elle bien Gouverner leurs Estats, \& parfaitement demesler les Interests de leurs Voisins ; ny leurs Generaux \& Officiers qui ont quelque commandement dans les Armées faire la Guerre avec succez. 
Les Gens d'Eglise sçavent assez de quelle Utilité elle leur est pour le Gouvernement Politique des choses Ecclésiastiques

Les Magistrats connoissent par elle l'Estenduë de leurs Jurisdictions.

Les gens de Finance ne s'en peuvent passer pour le Impositions, \& les Receptes des Deniers.

Les Négotians ne feroient leur Commerce qu'avec désavantage, si elle ne les instruisoit des Routes qu'il leur faut tenir.

Les Voyageurs qui n'entreprennent leurs Voyages que par curiosité ne pourroient reüssir dans leur dessein, s'ils n'estoient conduits par elle.

Et ceux-là mesme qui n'ont aucune Teinture de belles Lettres, n'y aucun Employ dans la Vie Civile, croiroient ne pouvoir passer agréablement, une Partie de leur grand loisir... (Sanson, 1682, Préface, 1-3, n.p.).

11 Parmi ceux qui sont dans la situation financière d'acheter des cartes, aucun ne semble avoir été oublié. Même ceux qui n'ont « aucune teinture de belles lettres » sont compris dans cet inventaire extensif.

Le quatrième ouvrage est la Méthode pour étudier la Géographie de Nicolas Lenglet du Fresnoy (1674-1755). Lenglet du Fresnoy, après avoir publié en 1713 une Méthode pour étudier l'histoire dans laquelle il avait placé un texte sur "l'étude de la Géographie », propose en 1716 une Méthode pour étudier la Géographie. Le livre va connaître une série de rééditions en 1736, 1741 et 1768. Le "Discours sur l'étude de la Géographie » (Lenglet du Fresnoy, 1716, t.1) court sur 137 pages et décrit la nécessité du savoir géographique, en le décomposant en 13 sous-titres. Les cinq premiers déclinent les composantes de son public. On y trouve ceux qui apprennent l'histoire distingués de ceux qui la lisent, puis viennent les guerriers, les politiques et les négociateurs, enfin ceux qui s'intéressent à la religion. Pour Lenglet, il est impossible de se passer de la géographie si l'on mène l'une de ces activités. Ainsi, pour ceux qui étudient l'histoire, faute de géographie "on tomberoit infailliblement dans les fautes qu'on reproche à quelques historiens". De même, pour les militaires, « la pratique de la guerre, nécessite une connaissance très détaillée du royaume \& en particulier de la Province qui sert de théâtre à la guerre ». Pour les politiques, la relation est directe : «Comme la bienséance règle la plupart des intérêts des Princes ; la situation des lieux, des villes, des provinces \& des Royaumes règle ces bienséances ». Quant au commerce c'est la navigation, décrite comme « une partie essentielle de la géographie », qui explique l'utilité de ce savoir. La religion, enfin, a besoin de comprendre la géographie pour comprendre les peuples, « car il ne saurait y avoir de convention générale et unanime » (Lenglet du Fresnoy, 1716, t. 1, ij, vj, vii, et xiij).

13 Un vaste public, liant savant et hommes d'action, administrateurs et militaires, voire commerçants composent le lectorat de la géographie selon ces divers auteurs. La répétition du discours, sans garantir l'efficacité participe cependant à mettre en place le lieu commun de l'utilité de la géographie. On retrouvera ces catégories dans de nombreux ouvrages de la seconde moitié du XVIII ${ }^{\mathrm{e}}$ siècle. Seule extension notable, en 1771, la Nouvelle méthode géographique de l'abbé Compan ajoute-t-il à ce public celui des femmes, cela non sans une supériorité masculine marquée :

Je ne crois pas qu'il soit nécessaire de s'étendre sur l'utilité que les femmes même peuvent retirer de l'étude de la Géographie. Et quelle science leur convient davantage? En avouant que l'éducation qu'elles reçoivent ne leur permet pas de s'élever à ces sciences abstraites, où le vol seul du génie peut conduire; ne leur faisons pas l'injustice de croire qu'elles ne puissent s'appliquer à rien de solide. Destinées à une vie sédentaire \& retirée, privées par conséquent de la ressource des voyages pénibles \& continus, qui, dans les hommes peuvent suppléer à l'étude de la 
géographie ; cette science seule peut les instruire sur les particularités du globe qu'elles habitent. (Compan, 1771, p. xxxj).

\section{La géographie dans un journal français, le cas du Mercure galant, puis français}

Le Mercure Galant, qui deviendra par la suite Mercure de France publie différents types de textes relatifs à la géographie. Dans certains cas, il ne s'agit que de simples publicités, dans d'autres on peut trouver des descriptions plus ou moins critiques des textes. Exceptionnellement, le journal peut être un lieu de controverse savante riche de réflexions propres à enrichir nos connaissances de la période (Verdier, 2018). Quoi qu'il en soit, l'essentiel des textes reprend les discours sur le public que nous avons évoqué à propos des livres. Ainsi, en 1706, à propos du Parfait géographe de Renaud Lecoq (Lecoq, 1701), le rédacteur du Mercure écrit

Il n'est pas seulement pour l'instruction de la jeunesse, \& comme il se trouve une infinité de gens, qui étant encore jeunes n'ont point appris la Géographie, ou à qui on ne l'a montrée que tres-superficiellement, leurs autres études ne leur permettant pas d'y employer beaucoup de temps, cette étude semble estre devenuë le partage des gens déjà en maturité, plutost que celle des écoliers. On peut même dire que les hommes en font une étude continuelle, la situation des affaires \& les guerres les engageant souvent à jetter les yeux sur leurs Cartes, \& à lire l'histoire des lieux où ces guerres sont allumées. (Mercure Galant, 1706/11,306-307).

Deux thèmes principaux sont développés, le premier à propos des militaires, dont on dit, par exemple en janvier 1731, que «le bréviaire d'un homme d'épée, c'est la Géographie, l'Histoire, les Fortifications, la Géométrie... » (Mercure Français, 1735/01, 79). L'idée est parfois un peu plus développée, comme dans le cas d'une "Lettre sur l'art militaire" d'octobre 1737 :

La Géographie sera-t-elle regardée comme superflue, pendant que la plupart des projets sont formés sur les Cartes des Pays où l'on fait la guerre. Plus la connoissance que l'on pouroit avoir, seroit parfaite, plus on trouveroit de facilité à dresser les Plans des marches d'armées, à déterminer la position des Camps, et à assurer les chemins qui y doivent conduire toutes sortes de provisions, lorsqu'ils sont établis; cela forme à la vérité une espèce de Géographie rapprochée, qui regarde particulièrement les Provinces Etrangères qui nous environnent, et celles de nos frontières qui les touchent ; car il n'est pas moins essentiel de les connoitre que celles de l'ennemi. (Mercure Français, 1737/10, 2193).

Le second thème est celui de l'apprentissage de l'histoire. Ainsi l'abbé Noblot écrit en juillet $1741:$ « Il est certain en effet que la Géographie \& la Chronologie sont comme les deux clefs de l'Histoire; l'une fixe le lieu, \& l'autre le tems des événemens » (Mercure Français 1741/07, 1520). On peut sur ce point trouver des morceaux de bravoure, comme un texte publié par le Mercure en mai 1751:

Ne connoissant pas, Monsieur, de Science plus curieuse que la Géographie, je crois entrer dans l'esprit de votre Journal, consacré à la curiosité en tout genre d'esprit, en vous communiquant mes vûes sur cette partie amusante de la Science Humaine. La Géographie est l'âme ou le corps, le grand Théâtre au moins de l'Histoire, de la double histoire même des hommes \& de la Nature. Elle est même, sans autre scène ni acteur, la propre Histoire de la Nature, servant par tout de Coryphée \& de modèle, autant que de scène à l'Histoire des hommes... (Mercure Français, 1751/05, p. 65). 
17 Mais l'essentiel se situe probablement ailleurs, d'une part dans l'association entre carte et géographie qui fait que le discours sur les usages de la carte s'étend à celui de la géographie. Là le discours est plus vaste, plus argumenté et reprend toute une série de lieux communs, comme celui sur la vue à distance que la carte rend possible. D'autre part dans l'aspect d'évidence qui est donné à la nécessité de la géographie. Ainsi, lorsque Le Rouge publie son atlas nouveau en 1748 (Le Rouge, 1748), il précise que ces cartes sont «dressées sur une échelle commode pour les officiers \& pour les Voyageurs » (Mercure Français, 1748/08, 162-163), rendant par là même l'usage évident.

Il reste que la différence entre les livres et ce journal ne sont que de détail. Au total donc, tout un discours qui met en place par la répétition une injonction géographique, tant au sein des ouvrages que dans la presse, faisant de la géographie un savoir utile et nécessaire à tous. Cette nécessité postulée se retrouve-t-elle dans la diffusion de ces livres?

\section{La géographie dans les bibliothèques privées}

Tenter d'avoir un aperçu de la diffusion des livres de géographie dans les bibliothèques privées reste malaisé. Les éléments dont nous disposons sont toujours en deçà de ce que nous souhaiterions. Les inventaires après décès font le plus souvent disparaître les volumes de peu de valeur, comme les octavo, alors que ceux-ci sont de plus en plus nombreux au XVIII ${ }^{e}$ siècle. Les inventaires des bibliothèques, composés lors de leurs ventes à l'encan, ne nous donnent que peu d'informations sur l'exhaustivité de ce qu'ils proposent. Combien d'ouvrages, usés, ou de valeur faible ont-ils était sortis de ces listes? Combien d'ouvrages de grande valeur sont-ils sortis avant que le marchandlibraire ne soit invité à venir examiner le fond? Sans parler de vol, on imagine aisément les achats à l'unité de volumes permettant de compléter la bibliothèque d'un proche des héritiers. La composition d'une bibliothèque au moment de sa vente a par ailleurs pour défaut d'arriver à la fin d'un processus. Peut-être qu'à un moment de nombreux livres de géographie furent achetés, et qu'ensuite, la connaissance acquise, il n'est plus paru nécessaire d'en acquérir d'autres. Une autre difficulté, toute aussi problématique réside dans la différence entre le fait d'avoir un livre dans sa bibliothèque et celui de l'avoir lu, voire de s'en servir. Cette question prend toute son ampleur avec la bibliophilie qui semble se développer à partir du XVII ${ }^{\mathrm{e}}$ siècle (Varry, 1997; Kenny, 2000). A ces aspects s'ajoute le fait qu'une bibliothèque n'a pas nécessairement la même durée de vie que son propriétaire. On l'a vu, elle peut être vendue du vivant de son propriétaire, et parfois en un seul lot. Mais elle peut aussi être héritée. Et les mélanges de bibliothèques peuvent avoir lieu à différents moments, ce qui peut, ou non, sous-entendre des tris. L'existence de doublons incite de ce point de vue à se poser des questions sur les origines des livres, voire, à une autre échelle, à penser les bibliothèques en tant que compositions.

Pour en finir avec ces difficultés, rien ne permet de garantir la représentativité d'un échantillon de catalogues de livres, dont les différents exemplaires sont arrivés jusqu'à nous sans que l'on sache réellement pourquoi. En effet, en dehors de la bibliophilie, à quoi sert de conserver ces ouvrages qui renvoient à des ventes passées, en dehors de conserver la trace d'un moment, ou la mémoire d'une bibliothèque et d'un personnage ? Le filtre de ces raisons, qui reste difficile à appréhender, doit donc être conservé à l'esprit lorsque l'on va analyser les contenus de ces ouvrages. Dans ce cas, 
l'échantillon se compose d'une cinquantaine de ces catalogues : 12 entre 1749 et 1758 , 23 entre 1759 et 1768,15 entre 1769 et 1788 et 5 entre 1789 et 1797 . Le contenu moyen de ces bibliothèques est de 4000 ouvrages, mais dans les faits les différences sont fortes. La plus petite bibliothèque est celle mise en vente lors du décès de Madame la Maréchale de Thomond en 1763. Elle compte 405 livres. La plus grosse est celle du Maréchal duc d'Estrées, mise en vente en 1740. Elle compte 20047 volumes. Au vue des premiers résultats, il n'est pas possible d'affirmer que la part que prennent les livres de géographie à l'intérieur des bibliothèques varie avec les dimensions de celles-ci. Autrement dit, il ne semble pas que chaque savoir soit au minimum représenté par un nombre d'ouvrages en-dessous duquel il n'est pas possible de descendre. Inversement, une grosse bibliothèque ne sous-entend en rien une grande collection d'ouvrages de géographie.

Que dire de la géographie dans ces catalogues ? Il convient en premier lieu de s'attacher à la géographie en tant que catégorie. De ce point de vue 52 des 53 collections traitées ont la même structure, ce qui semble souvent être due à une mise en ordre par un marchand-libraire. Guillaume de Bure aime ainsi rappeler dans chacun de ses ouvrages le classement qu'il a effectué de façon à rendre la vente possible. L'appellation est la division en «facultés » ${ }^{1}$ du catalogue. Selon ce classement, on a 5 catégories : Théologie, Jurisprudence, Sciences et arts, belles lettres et Histoire. La catégorie Histoire commence par une sous-catégorie Géographie. Il peut arriver que certains ouvrages ayant dans leur titre le mot "géographie » se retrouvent en dehors de la catégorie géographie, mais c'est assez rare.

Sur la période, la géographie représente un peu moins de $10 \%$ de l'histoire. Mais cette moyenne correspond dans les faits une évolution. En effet, alors que la moyenne est d'environ $6 \%$ entre 1729 et 1748 , elle tend à augmenter ensuite, autour de $9 \%$ entre 1749 et 1788 . La période suivante s'appuie pour l'instant sur trop peu de données pour que la moyenne de $25 \%$ nous apparaisse très fiable. Quoi qu'il en soit, dans la relation entre histoire et géographie, l'histoire prend clairement la part du lion, mais la part de la géographie connaît une croissance réelle.

La catégorie Géographie peut être décomposée, dans les descriptions les plus courantes, en géographie et en voyages. Parfois des sous-catégories comme "Géographie ancienne », ou "descriptions », qui correspondent le plus souvent aux atlas, voire aux «cartes » apparaissent. Lorsque les voyages sont nombreux (plus d'une cinquantaine), le classement opéré propose des sous-catégories soit, du plus proche au plus lointain, soit par continent. On trouve en outre, de façon récurrente, la mention de voyages imaginaires (rarement plus d'une dizaine). La relation entre la géographie et les voyages n'est que rarement explicitée, mais le cas du catalogue de la bibliothèque du Maréchal d'Estrées montre, en 1740, que celle-ci est d'échelle, un peu comme le passage de la géographie à la chorographie dans le cadre de la succession qui va de la cosmographie à la topographie. Ainsi dans le cas de cette bibliothèque, on passe de la Cosmographie) (10 ouvrages) à la «Description générale des différentes parties du monde » (6 ouvrages), à la "Description partielle des royaumes et provinces ", avec comme premier sous-titre "Voyages ». Chez l'Abbé Favier, qui possède jusqu'ici la bibliothèque la mieux fournie en livres de géographie ( 96 volumes et 149 si l'on y ajoute les voyages), l'ordre suivi est le même que dans la bibliothèque du Maréchal d'Estrées, mais sans qu'à aucun moment les catégories ne soient explicitées. 
tiennent, nous n'en prendrons ici qu'un. Il s'agit des Conseils pour former une bibliothèque peu nombreuse et choisie que Formey (1711-1797; voir le Dictionnaire des Journalistes) enseignant d'éloquence au Collège français de Berlin - fait paraître en 1755 (avec des rééditions en 1756, 1764, 1766 et 1771). L'ouvrage fourni 12 «classes de livres », ce qui décompose les 5 classes évoquées précédemment. Il offre à la géographie et aux voyages une classe à part entière. Sur la liste de 1700 références choisies dans la troisième édition de 1764 (le projet était à l'origine une bibliothèque de 100 livres) on en trouve 9 pour la géographie strictement dite, auquel (il ajoute mais sans les compter, au moins 200 cartes, parfois en atlas) et 18 pour les voyages auxquels Formey ajoute 9 livres pour l'héraldique qu'il adjoint à la géographie, soit 36 livres sur 1700 . On a donc $0.55 \%$ des livres décrits qui correspondent à la géographie et si on lui adjoint l'héraldique et les voyages, on a environ $2.1 \%$ du total. On n'est pas loin ici des 0.6 et 2.6 \% évoqués plus haut à propos des bibliothèques privées. Cela rapproche en outre les ouvrages de ces bibliothèques privées d'ouvrages utiles et non de simples faire valoir de leurs propriétaires.

Malgré ses faiblesses, cette série de catalogues peut donc, pour l'instant, être considérée comme étant représentative de la morphologie des bibliothèques de l'époque. Cela mène à une conclusion en demi-teinte. On peut en effet insister sur l'idée 
que la part de la géographie dans les bibliothèques est particulièrement modeste à une époque où les auteurs qui se revendiquent de ce savoir prétendent à une utilité quasiuniverselle. Mais on peut également insister, d'autre part, sur le fait que la géographie est dix fois plus présente dans les bibliothèques privées qu'elle ne l'est dans le catalogue de la Bibliothèque nationale. Sans donner trop de crédit à l'efficacité du discours des auteurs de textes géographiques, on peut, a minima, y voir un savoir qui s'affirme bien au-delà de ce que les indices relatifs à la production laissaient entendre.

Que tirer de cet ensemble d'observations effectuées selon des angles différents, avec des méthodes différentes? D'une part qu'il existe une tension entre ce que l'on peut observer du point de vue de la croissance de la publication de livres de géographie, de celui du discours conquérant des auteurs, et des indices matériels de la diffusion. Il est intéressant, d'autre part, de noter que le processus de diffusion s'effectue au moment où un autre, plus fort, concerne la cartographie. En effet, les cartes, qui sont de plus en plus intimement liées à la géographie se diffusent alors dans les divers savoirs de l'époque (Verdier, 2015a). L'évolution de la proportion des livres contenant des cartes durant la même période en offre un indice. Ils passent ainsi de $1,5 \%$ à $6,3 \%$ de l'ensemble des ouvrages publiés en français. En ajoutant les livres de géographie sans carte, on passerait de $2 \%$ à $8 \%$ durant cette période. Bilan plus favorable que celui qui a été évoqué à propos de ce vaste catalogue. L'enregistrement souvent réduit des cartes dans les catalogues de ventes de bibliothèques limite, hélas, l'évaluation qu'il aurait été possible de tenter pour savoir si la carte prenait là aussi un essor plus net qu'au sein des catalogues de la Bibliothèque nationale.

C'est dans cette force relative qui réside finalement la plus grande difficulté pour la géographie. En effet, la diffusion de la géographie bien au-delà de ses auteurs aurait pu laisser imaginer une institutionnalisation rapide du savoir. Or il n'en est rien, et il faudra attendre les années 1890 pour que la discipline géographique ne s'impose dans l'université française. Cela incite à tenter de comprendre ce qui a limité l'affirmation de ce savoir dans les années 1830-1890.

\section{BIBLIOGRAPHIE}

Balayé S., 1988, La Bibliothèque Nationale des origines à 1800, Genève, Droz.

Bayle P., 1704, Dictionnaire historique et critique, Rotterdam, Reinier Leers, t. 3, p. 2794.

Bourdieu P., 1997, Les usages sociaux de la science : pour une sociologie clinique du champ scientifique, Paris, Inra.

Broc N., 1970, « Une affaire de plagiat cartographique sous Louis XIV : le procès Delisle-Nolin », Revue d'histoire des Sciences et de leurs applications, vol. 23-2, p. 141-153.

Chartier R., 1992, L'ordre des livres. Lecteurs, auteurs, bibliothèques en Europe entre XVI ${ }^{e}$ et XVIII ${ }^{e}$ siècle, Aix en Provence, Alinéa. 
Chervel A., 1988, «L'histoire des disciplines scolaires. Réflexions sur un domaine de recherche », Histoire de l'éducation, $\mathrm{n}^{\circ}$ 38, p. 59-119.

Compan (Abbé), 1771, Nouvelle méthode géographique, précédée d'un traité de la Sphère \& des éléments de Géométrie, \& terminée par une géographie sacrée ; avec un discours préliminaire sur l'origine \& l'utilité de cette science, \& une table des longitudes \& latitudes des principales villes du Monde, conforme aux dernières Observations de MM. de l'Académie des Sciences, \& autres Astronomes, dédié à Madame, Paris, chez Mérigot jeune.

Dainville F. de, 1964, Le langage des géographes ; termes, signes, couleurs des cartes anciennes (1500-1800), Paris, A. et J. Picard.

Dictionnaire des journalistes (1600-1789), 2015-2019, Édition électronique revue, corrigée et augmentée, Voltaire Foundation, IHRIM UMR 5317, MSH-LSE USR 2005, http://dictionnairejournalistes.gazettes18e.fr/journaliste/310-jean-henri-formey, consulté le 28 septembre 2020.

Edgerton D., 1998, « De l'innovation aux usages. Dix thèses éclectiques sur l'histoire des techniques », Annales HES, n 4-5, p. 815-837.

Formey J. H. S., 1756, Conseils pour former une bibliothèque peu nombreuse mais choisie, Berlin, Chez Haude et Spener.

Hurtaut P.-T.-N., 1695, «Éloge du Père Augustin Lubin, Religieux de l'ordre de Saint Augustin », Journal des Sçavans, t. 23, p. 220-222.

Hurtaut P.-T.-N., 1779, Dictionnaire historique de la Ville de Paris et de ses environs, Paris, Moutard.

Kenny N., 2000, « Books in Space and Time : Bibliomania and Early Modern Histories of Learning and "Literature" in France », Modern Language Quaterly, vol. 61-2, p. 253-286.

Launay G. (de), 1681, La cosmographie aisée contenant la sphere, l'usage du Globe terrestre \& la Geographie, Paris, Estienne Michallet.

Le Rouge G.-L., 1748, Atlas nouveau portatif à l'usage des militaires, et du voyageur, contenant 91 cartes dressées sur les nouvelles observations, dédié à Monsieur le Comte de Maurepas, Ministre, Secrétaire d'Etat, par son très-humble \& très obéissant serviteur G. le Rouge, Paris, Chez G. Le Rouge.

Lecoq R., 1701, Le Parfait géographe, ou l'art d'apprendre aisément la géographie et l'histoire, Paris, chez Imbert de Bats.

Lenglet du Fresnoy N., 1716, Méthode pour étudier la géographie dans laquelle on donne une Description exacte de l'Univers, tirée des meilleurs Auteurs, \& formée sur les Observations de Messieurs de l'Académie Royale des Sciences, avec un Discours préliminaire sur l'étude de cette science, \& un catalogue des Cartes Géographiques, des relations, Voyages, \& Descriptions les plus nécessaires pour la Géographie, Paris, Charles Etienne Hochereau.

Lubin Augustin, 1678, Mercure Géographique ou le Guide du Curieux des Cartes Géographiques, Paris, Christophe Remy.

Martin H.-J., 1983, « Une croissance séculaire », Martin Henri-Jean (dir), Histoire de l'édition française : Le livre triomphant : 1660-1830, Paris, Promodis, p. 94-103.

Martin H.-J., et Chartier R. (dir.), 1982-1986, Histoire de l'édition française, Paris, Promodis, 4 vol. Mercure Galant, 1706/11, 1735/01, 1737/10, 1741/07, 1748/08, Lyon, chez Thomas Amaulry. Nordman D., 1998, « La géographie œil de l'histoire », EspaceTemps, n 66-67, p. 44-54.

Rey R., 1994, « La classification des sciences (1750-1850) », Revue de Synthèse, IV ${ }^{\mathrm{e}}$ série, n 1-2, p. 5-12. 
Rhein C., 1982, « La géographie, discipline scolaire et/ou science sociale ? (1860-1920) », Revue française de sociologie, vol. 23-2, p. 223-251.

Richelet P., 1680, Dictionnaire français contenant les mots et les choses, plusieurs nouvelles remarques sur la langue française... avec les termes les plus connus des arts et des sciences..., Genève, J.-H. Wider.

Sanson G., 1681, Introduction à la Géographie, Paris, Sanson Guillaume.

Varry D., 1997, « La collection de livres rares en France 1725-1939 », Bulletin des bibliothèques de France, $\mathrm{n}^{\circ} 3$, p. 90-91, en ligne : http://bbf.enssib.fr/consulter/bbf-1997-03-0090-010, consulté le 29 septembre 2020 .

Verdier N., 2018, "Entre publicité, débat scientifique et vulgarisation : Jean-Baptiste d'Anville et les journaux savants", in Hofmann Catherine et Haguet Lucile (dir.), Jean-Baptiste d'Anville, un cabinet savant au siècle des Lumières, Oxford/Paris, Oxford University Studies in the Enlightenment/BNF, pp. 237-260.

Verdier N., 2015a, « Entre diffusion de la carte et affirmation des savoirs géographiques en France : les paradoxes de la mise en place de la carte géographique au XVIII ${ }^{\mathrm{e}}$ siècle ", L'espace géographique, $\mathrm{n}^{\circ} 1$, p. 38-56.

Verdier N., 2015b, La carte avant les cartographes, l'avènement du régime cartographique en France au

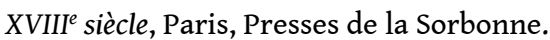

\section{NOTES}

1. «Faculté, [...] il se dit des différens corps qui composent une université. Il y a dans l'université de Paris quatre facultés ; celle des Arts, celle de Medecine, celle de Jurisprudence, \& celle de Theologie. Voyez les articles Université, Nation, Docteur, Bachelier, Licencié, Maître - ès - arts, Gradué, \&c.».", in Diderot Denis \& le Rond d'Alembert, Jean (dir.), L'Encyclopédie ou dictionnaire raisonné des sciences et des arts et des métiers par une société de gens de lettres, Paris, Briasson, David, Le breton, Durand, 1756, t.. 6, p. 371.

\section{RÉSUMÉS}

Définir un lectorat pour le XVIII ${ }^{\mathrm{e}}$ siècle est un problème insoluble. En effet, les tirages ne sont que rarement connus et une étude fine des bibliothèques privées sous-entendrait un immense travail de collecte dans les inventaires après-décès, sans que le résultat ne soit garanti. Nous essayerons donc dans cette présentation d'évaluer ce monde des lecteurs à partir de plusieurs indices. Les premiers seront statistiques, et tenteront d'évaluer la production d'ouvrages pour la période. Il s'agira ensuite d'aller rechercher du côté du lectorat que ces livres s'attribuent. En effet, certains ouvrages, comme l'Introduction à la Géographie des frères Sanson - de nombreuses fois rééditée tentent de dresser la liste de ceux à qui leur ouvrage s'adresse. Les suivants seront à rechercher dans les annonces contenues dans la presse de l'époque. Comment les ouvrages de géographie y sont-ils décrits et à qui sont-ils recommandés ? On évoquera pour finir la place des ouvrages de géographie dans quelques grandes bibliothèques privées, non pour y déterminer si la géographie 
est présente, elle l'est toujours, mais plus simplement pour en mesurer le poids par rapport aux autres savoirs.

Defining a readership for the 18th century is an intractable problem. In fact, print runs are only rarely known and a detailed study of private libraries would imply an immense amount of work in collecting in post-death inventories, without any guarantee of the result. We will therefore try in this presentation to evaluate this world of readers based on several clues. The first will be statistical, and will attempt to evaluate the production of books for the period. The second will be to look at the readership that these books are attributed to. Indeed, some books, such as the Introduction à la Géographie by the Sanson brothers - many times republished - attempt to list those to whom their work is addressed. The following ones are to be found in the announcements contained in the newspapers of the time. How are geography books described in them and to whom are they recommended? Finally, we will discuss the place of geography books in some large private libraries, not to determine whether geography is present there, it is always present, but more simply to measure its weight in relation to other knowledge.

\section{INDEX}

Mots-clés : Livre de géographie, lectorat, édition, publicité, bibliothèques

Keywords : Geography books, readership, publishing, advertising, libraries

\section{AUTEUR}

\section{NICOLAS VERDIER}

Directeur de recherche au CNRS, Directeur d'Etude à l'EHESS, co-directeur de l'Equipe d'Epistémologie et d'Histoire de la Géographie (EHGo). Adresse personnelle : 25, route de Gallerie, 41700, Cour-Cheverny. Adresse professionnelle : UMR 8504 Géographie-cité, Campus Condorcet, 5, cours des Humanités, 93322 Aubervilliers cedex. nicolas.verdier@parisgeo.cnrs.fr 